\title{
Subelliptic geometric Hardy type inequalities on half-spaces and convex domains
}

\section{Michael Ruzhansky ${ }^{1,2} \cdot$ Bolys Sabitbek ${ }^{2,3} \cdot$ Durvudkhan Suragan $^{4}$}

Received: 6 November 2019 / Accepted: 26 February 2020 / Published online: 2 April 2020

(c) The Author(s) 2020

\begin{abstract}
In this paper we present $L^{2}$ and $L^{p}$ versions of the geometric Hardy inequalities in half-spaces and convex domains on stratified (Lie) groups. As a consequence, we obtain the geometric uncertainty principles. We give examples of the obtained results for the Heisenberg and the Engel groups.
\end{abstract}

Keywords Stratified groups · Geometric Hardy inequality $\cdot$ Half-space $\cdot$ Convex domain

Mathematics Subject Classification $35 \mathrm{~A} 23 \cdot 35 \mathrm{H} 20$

\section{Introduction}

In the Euclidean setting, a geometric Hardy inequality in a (Euclidean) convex domain $\Omega$ has the following form

Communicated by Joachim Toft.

Bolys Sabitbek

b.sabitbek@math.kz

Michael Ruzhansky

Michael.Ruzhansky@ugent.be

Durvudkhan Suragan

durvudkhan.suragan@nu.edu.kz

1 Department of Mathematics: Analysis, Logic and Discrete Mathematics, Ghent University, Ghent, Belgium

2 School of Mathematical Sciences, Queen Mary University of London, London, UK

3 Institute of Mathematics and Mathematical Modeling, Al-Farabi Kazakh National University, Almaty, Kazakhstan

4 Department of Mathematics, School of Sciences and Humanities, Nazarbayev University, 53 Kabanbay Batyr Ave, 010000 Nursultan, Kazakhstan 


$$
\int_{\Omega}|\nabla u|^{2} d x \geq \frac{1}{4} \int_{\Omega} \frac{|u|^{2}}{\operatorname{dist}(x, \partial \Omega)^{2}} d x,
$$

for $u \in C_{0}^{\infty}(\Omega)$ with the sharp constant $1 / 4$. There is a number of studies related to this subject, see e.g. [1-3, 5, 6, 13].

In the case of the Heisenberg group $\mathbb{H}$, Luan and Yang [12] obtained the following Hardy inequality on the half space $\mathbb{W}^{+}:=\left\{\left(x_{1}, x_{2}, x_{3}\right) \in \mathbb{W}, \mid x_{3}>0\right\}$ for $u \in C_{0}^{\infty}\left(\mathbb{T}^{+}\right)$

$$
\int_{\mathbb{H}^{+}}\left|\nabla_{\mathbb{H}} u\right|^{2} d x \geq \int_{\mathbb{H}^{+}} \frac{\left|x_{1}\right|^{2}+\left|x_{2}\right|^{2}}{x_{3}^{2}}|u|^{2} d x .
$$

Moreover, the geometric $L^{p}$-Hardy inequalities for the sub-Laplacian on the convex domain in the Heisenberg group was obtained by Larson [11] which also generalises the previous result in [12]. In this note by using the approach in [11] we obtain the geometric Hardy type inequalities on the half-spaces and the convex domains on general stratified groups, so our results extend known results of Abelian (Euclidean) and Heisenberg groups.

Thus, the main aim of this paper is to prove the geometric Hardy type inequalities on general stratified groups. As consequences, the geometric uncertainty principles are obtained. In Sect. 2 we present $L^{2}$ and $L^{p}$ versions of the subelliptic geometric Hardy type inequalities on the half-space. In Sect. 3, we show subelliptic $L^{2}$ and $L^{p}$ versions of the geometric Hardy type inequalities on the convex domains.

\subsection{Preliminaries}

Let $\mathbb{G}=\left(\mathbb{R}^{n}, \circ, \delta_{\lambda}\right)$ be a stratified Lie group (or a homogeneous Carnot group), with dilation structure $\delta_{\lambda}$ and Jacobian generators $X_{1}, \ldots, X_{N}$, so that $N$ is the dimension of the first stratum of $\mathbb{G}$. We denote by $Q$ the homogeneous dimension of $\mathbb{G}$. We refer to [9], or to the recent books $[4,8]$ for extensive discussions of stratified Lie groups and their properties.

The sub-Laplacian on $\mathbb{G}$ is given by

$$
\mathcal{L}=\sum_{k=1}^{N} X_{k}^{2}
$$

We also recall that the standard Lebesque measure $d x$ on $\mathbb{R}^{n}$ is the Haar measure for $\mathbb{G}$ (see, e.g. [8, Proposition 1.6.6]). Each left invariant vector field $X_{k}$ has an explicit form and satisfies the divergence theorem, see e.g. [8] for the derivation of the exact formula: more precisely, we can formulate

$$
X_{k}=\frac{\partial}{\partial x_{k}^{\prime}}+\sum_{l=2}^{r} \sum_{m=1}^{N_{l}} a_{k, m}^{(l)}\left(x^{\prime}, \ldots, x^{(l-1)}\right) \frac{\partial}{\partial x_{m}^{(l)}},
$$

with $x=\left(x^{\prime}, x^{(2)}, \ldots, x^{(r)}\right)$, where $r$ is the step of $\mathbb{G}$ and $x^{(l)}=\left(x_{1}^{(l)}, \ldots, x_{N_{l}}^{(l)}\right)$ are the variables in the $l^{\text {th }}$ stratum, see also [8, Section 3.1.5] for a general presentation. The horizontal gradient is given by 


$$
\nabla_{\mathbb{G}}:=\left(X_{1}, \ldots, X_{N}\right),
$$

and the horizontal divergence is defined by

$$
\operatorname{div}_{\mathbb{G}} v:=\nabla_{\mathbb{G}} \cdot v .
$$

We now recall the divergence formula in the form of [14, Proposition 3.1]. Let $f_{k} \in C^{1}(\Omega) \bigcap C(\bar{\Omega}), k=1, \ldots, N$. Then for each $k=1, \ldots, N$, we have

$$
\int_{\Omega} X_{k} f_{k} d z=\int_{\partial \Omega} f_{k}\left\langle X_{k}, d z\right\rangle
$$

Consequently, we also have

$$
\int_{\Omega} \sum_{k=1}^{N} X_{k} f_{k} d z=\int_{\partial \Omega} \sum_{k=1}^{N} f_{k}\left\langle X_{k}, d z\right\rangle .
$$

\section{Hardy type inequalities on half-space}

\section{1 $L^{2}$-Hardy inequality on the half-space of $\mathbb{G}$}

In this section we present the geometric $L^{2}$-Hardy inequality on the half-space of $\mathbb{G}$. We define the half-space as follows

$$
\mathbb{G}^{+}:=\{x \in \mathbb{G}:\langle x, v\rangle>d\},
$$

where $v:=\left(v_{1}, \ldots, v_{r}\right)$ with $v_{j} \in \mathbb{R}^{N_{j}}, j=1, \ldots, r$, is the Riemannian outer unit normal to $\partial \mathbb{G}^{+}$(see [10]) and $d \in \mathbb{R}$. The Euclidean distance to the boundary $\partial \mathbb{G}^{+}$is denoted by $\operatorname{dist}\left(x, \partial \mathbb{G}^{+}\right)$and defined as follows

$$
\operatorname{dist}\left(x, \partial \mathbb{G}^{+}\right)=\langle x, v\rangle-d .
$$

Moreover, there is an angle function on $\partial \mathbb{G}^{+}$which is defined by Garofalo in [10] as

$$
\mathcal{W}(x)=\sqrt{\sum_{i=1}^{N}\left\langle X_{i}(x), v\right\rangle^{2}} .
$$

Theorem 2.1 Let $\mathbb{G}^{+}$be a half-space of a stratified group $\mathbb{G}$. Then for all $\beta \in \mathbb{R}$ we have

$$
\begin{aligned}
\int_{\mathbb{G}^{+}}\left|\nabla_{\mathbb{G}} u\right|^{2} d x \geq & C_{1}(\beta) \int_{\mathbb{G}^{+}} \frac{\mathcal{W}(x)^{2}}{\operatorname{dist}\left(x, \partial \mathbb{G}^{+}\right)^{2}}|u|^{2} d x \\
& +\beta \int_{\mathbb{G}^{+}} \sum_{i=1}^{N} \frac{X_{i}\left\langle X_{i}(x), v\right\rangle}{\operatorname{dist}\left(x, \partial \mathbb{G}^{+}\right)}|u|^{2} d x,
\end{aligned}
$$

Birkhäuser 
for all $u \in C_{0}^{\infty}\left(\mathbb{G}^{+}\right)$and where $C_{1}(\beta):=-\left(\beta^{2}+\beta\right)$.

Remark 2.2 If $\mathbb{G}$ has step $r=2$, then for $i=1, \ldots, N$ we have the following leftinvariant vector fields

$$
X_{i}=\frac{\partial}{\partial x_{i}^{\prime}}+\sum_{s=1}^{N_{2}} \sum_{m=1}^{N} a_{m, i}^{s} x_{m}^{\prime} \frac{\partial}{\partial x_{s}^{\prime \prime}},
$$

where $a_{m, i}^{s}$ are the group constants (see, e.g. [7, Formula (2.14)] for the definition). Also we have $x:=\left(x^{\prime}, x^{\prime \prime}\right)$ with $x^{\prime}=\left(x_{1}^{\prime}, \ldots, x_{N}^{\prime}\right), x^{\prime \prime}=\left(x_{1}^{\prime \prime}, \ldots, x_{N_{2}}^{\prime \prime}\right)$, and also $v:=\left(v^{\prime}, v^{\prime \prime}\right)$ with $v^{\prime}=\left(v_{1}^{\prime}, \ldots, v_{N}^{\prime}\right)$ and $v^{\prime \prime}=\left(v_{1}^{\prime \prime}, \ldots, v_{N_{2}}^{\prime \prime}\right)$.

Corollary 2.3 Let $\mathbb{G}^{+}$be a half-space of a stratified group $\mathbb{G}$ of step $r=2$. For all $\beta \in \mathbb{R}$ and $u \in C_{0}^{\infty}\left(\mathbb{G}^{+}\right)$we have

$$
\begin{aligned}
\int_{\mathbb{G}^{+}}\left|\nabla_{\mathbb{G}} u\right|^{2} d x \geq & C_{1}(\beta) \int_{\mathbb{G}^{+}} \frac{\mathcal{W}(x)^{2}}{\operatorname{dist}\left(x, \partial \mathbb{G}^{+}\right)^{2}}|u|^{2} d x \\
& +K(a, v, \beta) \int_{\mathbb{G}^{+}} \frac{|u|^{2}}{\operatorname{dist}\left(x, \partial \mathbb{G}^{+}\right)} d x,
\end{aligned}
$$

where $C_{1}(\beta):=-\left(\beta^{2}+\beta\right)$ and $K(a, v, \beta):=\beta \sum_{s=1}^{N_{2}} \sum_{i=1}^{N} a_{i, i}^{s} \nu_{s}^{\prime \prime}$.

Proof of Theorem 2.1 To prove inequality (2.3) we use the method of factorization. Thus, for any $W:=\left(W_{1}, \ldots, W_{N}\right), W_{i} \in C^{1}\left(\mathbb{G}^{+}\right)$real-valued, which will be chosen later, by a simple computation we have

$$
\begin{aligned}
0 \leq \int_{\mathbb{G}^{+}}\left|\nabla_{\mathbb{G}} u+\beta W u\right|^{2} d x & =\int_{\mathbb{G}^{+}}\left|\left(X_{1} u, \ldots, X_{N} u\right)+\beta\left(W_{1}, \ldots, W_{N}\right) u\right|^{2} d x \\
& =\int_{\mathbb{G}^{+}}\left|\left(X_{1} u+\beta W_{1} u, \ldots, X_{N} u+\beta W_{N} u\right)\right|^{2} d x \\
& =\int_{\mathbb{G}^{+}} \sum_{i=1}^{N}\left|X_{i} u+\beta W_{i} u\right|^{2} d x \\
& =\int_{\mathbb{G}^{+}} \sum_{i=1}^{N}\left[\left|X_{i} u\right|^{2}+2 \operatorname{Re} \beta W_{i} u X_{i} u+\beta^{2} W_{i}^{2}|u|^{2}\right] d x \\
& =\int_{\mathbb{G}^{+}} \sum_{i=1}^{N}\left[\left|X_{i} u\right|^{2}+\beta W_{i} X_{i}|u|^{2}+\beta^{2} W_{i}^{2}|u|^{2}\right] d x \\
& =\int_{\mathbb{G}^{+}} \sum_{i=1}^{N}\left[\left|X_{i} u\right|^{2}-\beta\left(X_{i} W_{i}\right)|u|^{2}+\beta^{2} W_{i}^{2}|u|^{2}\right] d x .
\end{aligned}
$$


From the above expression we get the inequality

$$
\int_{\mathbb{G}^{+}}\left|\nabla_{\mathbb{G}} u\right|^{2} d x \geq \int_{\mathbb{G}^{+}} \sum_{i=1}^{N}\left[\left(\beta\left(X_{i} W_{i}\right)-\beta^{2} W_{i}^{2}\right)|u|^{2}\right] d x .
$$

Let us now take $W_{i}$ in the form

$$
W_{i}(x)=\frac{\left\langle X_{i}(x), v\right\rangle}{\operatorname{dist}\left(x, \partial \mathbb{G}^{+}\right)}=\frac{\left\langle X_{i}(x), v\right\rangle}{\langle x, v\rangle-d},
$$

where

$$
X_{i}(x)=(\overbrace{0, \ldots, 1}^{i}, \ldots, 0, a_{i, 1}^{(2)}\left(x^{\prime}\right), \ldots, a_{i, N_{r}}^{(r)}\left(x^{\prime}, x^{(2)}, \ldots, x^{(r-1)}\right)),
$$

and

$$
v=\left(v_{1}, v_{2}, \ldots, v_{r}\right), \quad v_{j} \in \mathbb{R}^{N_{j}} .
$$

Now $W_{i}(x)$ can be written as

$$
W_{i}(x)=\frac{v_{1, i}+\sum_{l=2}^{r} \sum_{m=1}^{N_{l}} a_{i, m}^{(l)}\left(x^{\prime}, \ldots, x^{(l-1)}\right) v_{l, m}}{\sum_{l=1}^{r} x^{(l)} \cdot v_{l}-d} .
$$

By a direct computation we have

$$
\begin{aligned}
X_{i} W_{i}(x) & =\frac{X_{i}\left\langle X_{i}(x), v\right\rangle \operatorname{dist}\left(x, \partial \mathbb{G}^{+}\right)-\left\langle X_{i}(x), v\right\rangle X_{i}\left(\operatorname{dist}\left(x, \partial \mathbb{G}^{+}\right)\right)}{\operatorname{dist}\left(x, \partial \mathbb{G}^{+}\right)^{2}} \\
& =\frac{X_{i}\left\langle X_{i}(x), v\right\rangle}{\operatorname{dist}\left(x, \partial \mathbb{G}^{+}\right)}-\frac{\left\langle X_{i}(x), v\right\rangle^{2}}{\operatorname{dist}\left(x, \partial \mathbb{G}^{+}\right)^{2}},
\end{aligned}
$$

where

$$
\begin{aligned}
X_{i}\left(\operatorname{dist}\left(x, \partial \mathbb{G}^{+}\right)\right) & =X_{i}\left(\sum_{k=1}^{N} x_{k}^{\prime} v_{1, k}+\sum_{l=2}^{r} \sum_{m=1}^{N_{l}} x_{m}^{(l)} v_{l, m}-d\right) \\
& =v_{1, i}+\sum_{l=2}^{r} \sum_{m=1}^{N_{l}} a_{i, m}^{(l)}\left(x^{\prime}, \ldots, x^{(l-1)}\right) v_{l, m} \\
& =\left\langle X_{i}(x), v\right\rangle .
\end{aligned}
$$

Inserting the expression (2.8) in (2.6) we get 


$$
\begin{aligned}
\int_{\mathbb{G}^{+}}\left|\nabla_{\mathbb{G}} u\right|^{2} d x \geq & -\left(\beta^{2}+\beta\right) \int_{\mathbb{G}^{+}} \sum_{i=1}^{N} \frac{\left\langle X_{i}(x), v\right\rangle^{2}}{\operatorname{dist}\left(x, \partial \mathbb{G}^{+}\right)^{2}}|u|^{2} d x \\
& +\beta \int_{\mathbb{G}^{+}} \sum_{i=1}^{N} \frac{X_{i}\left\langle X_{i}(x), v\right\rangle}{\operatorname{dist}\left(x, \partial \mathbb{G}^{+}\right)}|u|^{2} d x .
\end{aligned}
$$

The proof of Theorem 2.1 is finished.

As consequences of Theorem 2.1, we have the geometric Hardy inequalities on the half-space without an angle function, which seems an interesting new result on $\mathbb{G}$.

Corollary 2.4 Let $\mathbb{G}^{+}$be a half-space of a stratified group $\mathbb{G}$. Then we have

$$
\int_{\mathbb{G}^{+}}\left|\nabla_{\mathbb{G}} u\right|^{2} d x \geq \frac{1}{4} \int_{\mathbb{G}^{+}} \frac{|u|^{2}}{\operatorname{dist}\left(x, \partial \mathbb{G}^{+}\right)^{2}} d x,
$$

for all $u \in C_{0}^{\infty}\left(\mathbb{G}^{+}\right)$.

Proof of Corollary 2.4 Let $x:=\left(x^{\prime}, x^{(2)}, \ldots, x^{(r)}\right) \in \mathbb{G}$ with $x^{\prime}=\left(x_{1}^{\prime}, \ldots, x_{N}^{\prime}\right)$ and $x^{(j)} \in \mathbb{R}^{N_{j}}, j=2, \ldots, r$. By taking $v:=\left(v^{\prime}, 0, \ldots, 0\right)$ with $v^{\prime}=\left(v_{1}^{\prime}, \ldots, v_{N}^{\prime}\right)$, we have that

$$
X_{i}(x)=(\overbrace{0, \ldots, 1}^{i}, \ldots, 0, a_{i, 1}^{(2)}\left(x^{\prime}\right), \ldots, a_{i, N_{r}}^{(r)}\left(x^{\prime}, x^{(2)}, \ldots, x^{(r-1)}\right))
$$

we have

$$
\sum_{i=1}^{N}\left\langle X_{i}(x), v\right\rangle^{2}=\sum_{i=1}^{N}\left(v_{i}^{\prime}\right)^{2}=\left|v^{\prime}\right|^{2}=1
$$

and

$$
X_{i}\left\langle X_{i}(x), v\right\rangle=X_{i} v_{i}^{\prime}=0
$$

Inserting the above expressions in inequality (2.3) we arrive at

$$
\int_{\mathbb{G}^{+}}\left|\nabla_{\mathbb{G}} u\right|^{2} d x \geq-\left(\beta^{2}+\beta\right) \int_{\mathbb{G}^{+}} \frac{|u|^{2}}{\operatorname{dist}\left(x, \partial \mathbb{G}^{+}\right)^{2}} d x .
$$

For optimisation we differentiate the right-hand side of integral with respect to $\beta$, then we have

$$
-2 \beta-1=0,
$$

which implies 


$$
\beta=-\frac{1}{2}
$$

We complete the proof.

We also have the geometric uncertainty principle on the half-space of $\mathbb{G}^{+}$.

Corollary 2.5 Let $\mathbb{G}^{+}$be a half-space of a stratified group $\mathbb{G}$. Then we have

$$
\left(\int_{\mathbb{G}^{+}}\left|\nabla_{\mathbb{G}} u\right|^{2} d x\right)^{\frac{1}{2}}\left(\int_{\mathbb{G}^{+}} \operatorname{dist}\left(x, \partial \mathbb{G}^{+}\right)^{2}|u|^{2} d x\right)^{\frac{1}{2}} \geq \frac{1}{2} \int_{\mathbb{G}^{+}}|u|^{2} d x
$$

for all $u \in C_{0}^{\infty}\left(\mathbb{G}^{+}\right)$.

Proof of Corollary 2.5 By using (2.9) and the Cauchy-Schwarz inequality we get

$$
\begin{aligned}
\int_{\mathbb{G}^{+}} & \left|\nabla_{\mathbb{G}} u\right|^{2} d x \int_{\mathbb{G}^{+}} \operatorname{dist}\left(x, \partial \mathbb{G}^{+}\right)^{2}|u|^{2} d x \\
& \geq \frac{1}{4} \int_{\mathbb{G}^{+}} \frac{1}{\operatorname{dist}\left(x, \partial \mathbb{G}^{+}\right)^{2}}|u|^{2} d x \int_{\mathbb{G}^{+}} \operatorname{dist}\left(x, \partial \mathbb{G}^{+}\right)^{2}|u|^{2} d x \\
& \geq \frac{1}{4}\left(\int_{\mathbb{G}^{+}}|u|^{2} d x\right)^{2} .
\end{aligned}
$$

To demonstrate our general result in a particular case, here we consider the Heisenberg group, which is a well-known example of step $r=2$ (stratified) group.

Corollary 2.6 Let $\mathbb{H}^{+}=\left\{\left(x_{1}, x_{2}, x_{3}\right) \in \mathbb{U} \mid x_{3}>0\right\}$ be a half-space of the Heisenberg group $\mathbb{H}$. Then for any $u \in C_{0}^{\infty}\left(\mathbb{H}^{+}\right)$we have

$$
\int_{\mathbb{H}^{+}}\left|\nabla_{\mathbb{H}} u\right|^{2} d x \geq \int_{\mathbb{M}^{+}} \frac{\left|x_{1}\right|^{2}+\left|x_{2}\right|^{2}}{x_{3}^{2}}|u|^{2} d x,
$$

where $\nabla_{\mathbb{W}}=\left\{X_{1}, X_{2}\right\}$.

Proof of Corollary 2.6 Recall that the left-invariant vector fields on the Heisenberg group are generated by the basis

$$
\begin{aligned}
& X_{1}=\frac{\partial}{\partial x_{1}}+2 x_{2} \frac{\partial}{\partial x_{3}} \\
& X_{2}=\frac{\partial}{\partial x_{2}}-2 x_{1} \frac{\partial}{\partial x_{3}},
\end{aligned}
$$

with the commutator 


$$
\left[X_{1}, X_{2}\right]=-4 \frac{\partial}{\partial x_{3}}
$$

For $x=\left(x_{1}, x_{2}, x_{3}\right)$, choosing $v=(0,0,1)$ as the unit vector in the direction of $x_{3}$ and taking $d=0$ in inequality (2.3), we get

$$
X_{1}(x)=\left(1,0,2 x_{2}\right) \quad \text { and } \quad X_{2}(x)=\left(0,1,-2 x_{1}\right),
$$

and

$$
\begin{aligned}
& \left\langle X_{1}(x), v\right\rangle=2 x_{2}, \quad \text { and } \quad\left\langle X_{2}(x), v\right\rangle=-2 x_{1}, \\
& X_{1}\left\langle X_{1}(x), v\right\rangle=0, \quad \text { and } \quad X_{2}\left\langle X_{2}(x), v\right\rangle=0 .
\end{aligned}
$$

Therefore, with $\mathcal{W}(x)$ as in (2.2), we have

$$
\frac{\mathcal{W}(x)^{2}}{\operatorname{dist}\left(x, \partial \mathbb{G}^{+}\right)^{2}}=4 \frac{\left|x_{1}\right|^{2}+\left|x_{2}\right|^{2}}{x_{3}^{2}} .
$$

Substituting these into inequality (2.3) we arrive at

$$
\int_{\mathbb{U}^{+}}\left|\nabla_{\mathbb{G}} u\right|^{2} d x \geq \int_{\mathbb{G}^{+}} \frac{\left|x_{1}\right|^{2}+\left|x_{2}\right|^{2}}{x_{3}^{2}}|u|^{2} d x,
$$

taking $\beta=-\frac{1}{2}$.

Let us present an example for the step $r=3$ (stratified) groups. A well-known stratified group with step three is the Engel group, which can be denoted by $\mathbb{E}$. Topologically $\mathbb{E}$ is $\mathbb{R}^{4}$ with the group law of $\mathbb{E}$, which is given by

$$
x \circ y=\left(x_{1}+y_{1}, x_{2}+y_{2}, x_{3}+y_{3}+P_{1}, x_{4}+y_{4}+P_{2}\right),
$$

where

$$
\begin{aligned}
& P_{1}=\frac{1}{2}\left(x_{1} y_{2}-x_{2} y_{1}\right), \\
& P_{2}=\frac{1}{2}\left(x_{1} y_{3}-x_{3} y_{1}\right)+\frac{1}{12}\left(x_{1}^{2} y_{2}-x_{1} y_{1}\left(x_{2}+y_{2}\right)+x_{2} y_{1}^{2}\right) .
\end{aligned}
$$

The left-invariant vector fields of $\mathbb{E}$ are generated by the basis

$$
\begin{aligned}
& X_{1}=\frac{\partial}{\partial x_{1}}-\frac{x_{2}}{2} \frac{\partial}{\partial x_{3}}-\left(\frac{x_{3}}{2}-\frac{x_{1} x_{2}}{12}\right) \frac{\partial}{\partial x_{4}} \\
& X_{2}=\frac{\partial}{\partial x_{2}}+\frac{x_{1}}{2} \frac{\partial}{\partial x_{3}}+\frac{x_{1}^{2}}{12} \frac{\partial}{\partial x_{4}} \\
& X_{3}=\frac{\partial}{\partial x_{3}}+\frac{x_{1}}{2} \frac{\partial}{\partial x_{4}} \\
& X_{4}=\frac{\partial}{\partial x_{4}}
\end{aligned}
$$


Corollary 2.7 Let $\mathbb{E}^{+}=\left\{x:=\left(x_{1}, x_{2}, x_{3}, x_{4}\right) \in \mathbb{E} \mid\langle x, v\rangle>0\right\}$ be a half-space of the Engel group $\mathbb{E}$. Then for all $\beta \in \mathbb{R}$ and $u \in C_{0}^{\infty}\left(\mathbb{E}^{+}\right)$we have

$$
\begin{aligned}
\int_{\mathbb{E}^{+}}\left|\nabla_{\mathbb{E}} u\right|^{2} d x \geq & C_{1}(\beta) \int_{\mathbb{E}^{+}} \frac{\left\langle X_{1}(x), v\right\rangle^{2}+\left\langle X_{2}(x), v\right\rangle^{2}}{\operatorname{dist}\left(x, \partial \mathbb{E}^{+}\right)^{2}}|u|^{2} d x \\
& +\frac{\beta}{3} \int_{\mathbb{E}^{+}} \frac{x_{2} v_{4}}{\operatorname{dist}\left(x, \partial \mathbb{E}^{+}\right)}|u|^{2} d x
\end{aligned}
$$

where $\nabla_{\mathbb{E}}=\left\{X_{1}, X_{2}\right\}, v:=\left(\nu_{1}, \nu_{2}, \nu_{3}, \nu_{4}\right)$, and $C_{1}(\beta)=-\left(\beta^{2}+\beta\right)$.

Remark 2.8 If we take $v_{4}=0$ in (2.12), then we have the following inequality on $\mathbb{E}$, by taking $\beta=-\frac{1}{2}$,

$$
\int_{\mathbb{E}^{+}}\left|\nabla_{\mathbb{E}} u\right|^{2} d x \geq \frac{1}{4} \int_{\mathbb{E}^{+}} \frac{\left\langle X_{1}(x), v\right\rangle^{2}+\left\langle X_{2}(x), v\right\rangle^{2}}{\operatorname{dist}\left(x, \partial \mathbb{E}^{+}\right)^{2}}|u|^{2} d x .
$$

Proof of Corollary 2.7 As we mentioned, the Engel group has the following basis of the left-invariant vector fields

$$
\begin{aligned}
& X_{1}=\frac{\partial}{\partial x_{1}}-\frac{x_{2}}{2} \frac{\partial}{\partial x_{3}}-\left(\frac{x_{3}}{2}-\frac{x_{1} x_{2}}{12}\right) \frac{\partial}{\partial x_{4}}, \\
& X_{2}=\frac{\partial}{\partial x_{2}}+\frac{x_{1}}{2} \frac{\partial}{\partial x_{3}}+\frac{x_{1}^{2}}{12} \frac{\partial}{\partial x_{4}}
\end{aligned}
$$

with the following two (non-zero) commutators

$$
\begin{aligned}
& X_{3}=\left[X_{1}, X_{2}\right]=\frac{\partial}{\partial x_{3}}+\frac{x_{1}}{2} \frac{\partial}{\partial x_{4}}, \\
& X_{4}=\left[X_{1}, X_{3}\right]=\frac{\partial}{\partial x_{4}} .
\end{aligned}
$$

Thus, we have

$$
\begin{aligned}
& X_{1}(x)=\left(1,0,-\frac{x_{2}}{2},-\left(\frac{x_{3}}{2}-\frac{x_{1} x_{2}}{12}\right)\right), \\
& X_{2}(x)=\left(0,1, \frac{x_{1}}{2}, \frac{x_{1}^{2}}{12}\right) .
\end{aligned}
$$

A direct calculation gives that

$$
\begin{aligned}
& \left\langle X_{1}(x), v\right\rangle=v_{1}-\frac{x_{2}}{2} v_{3}-\left(\frac{x_{3}}{2}-\frac{x_{1} x_{2}}{12}\right) v_{4}, \\
& \left\langle X_{2}(x), v\right\rangle=v_{2}+\frac{x_{1}}{2} v_{3}+\frac{x_{1}^{2}}{12} v_{4}, \\
& X_{1}\left\langle X_{1}(x), v\right\rangle=\frac{x_{2}}{12} v_{4}+\frac{x_{2}}{4} v_{4}=\frac{x_{2} v_{4}}{3}, \\
& X_{2}\left\langle X_{2}(x), v\right\rangle=0 .
\end{aligned}
$$


Now substituting these into inequality (2.3) we obtain the desired result.

\section{$2.2 L^{p}$-Hardy inequality on $\mathbb{G}^{+}$}

Here we construct an $L^{p}$ version of the geometric Hardy inequality on the half-space of $\mathbb{G}$ as a generalisation of the previous theorem. We define the $p$-version of the angle function by $\mathcal{W}_{p}$, which is given by the formula

$$
\mathcal{W}_{p}(x)=\left(\sum_{i=1}^{N}\left|\left\langle X_{i}(x), v\right\rangle\right|^{p}\right)^{\frac{1}{p}}
$$

Theorem 2.9 Let $\mathbb{G}^{+}$be a half-space of a stratified group $\mathbb{G}$. Then for all $\beta \in \mathbb{R}$ we have

$$
\begin{aligned}
\int_{\mathbb{G}^{+}} \sum_{i=1}^{N}\left|X_{i} u\right|^{p} d x \geq & C_{2}(\beta, p) \int_{\mathbb{G}^{+}} \frac{\mathcal{W}_{p}(x)^{p}}{\operatorname{dist}\left(x, \partial \mathbb{G}^{+}\right)^{p}}|u|^{p} d x \\
& +\beta(p-1) \int_{\mathbb{G}^{+}} \sum_{i=1}^{N}\left(\frac{\left|\left\langle X_{i}(x), v\right\rangle\right|}{\operatorname{dist}\left(x, \partial \mathbb{G}^{+}\right)}\right)^{p-2} \frac{X_{i}\left\langle X_{i}(x), v\right\rangle}{\operatorname{dist}\left(x, \partial \mathbb{G}^{+}\right)}|u|^{p} d x
\end{aligned}
$$

for all $u \in C_{0}^{\infty}\left(\mathbb{G}^{+}\right), 1<p<\infty$ and $C_{2}(\beta, p):=-(p-1)\left(|\beta|^{\frac{p}{p-1}}+\beta\right)$.

Proof of Theorem 2.9 We use the standard method such as the divergence theorem to obtain the inequality (2.14). For $W \in C^{\infty}\left(\mathbb{G}^{+}\right)$and $f \in C^{1}\left(\mathbb{G}^{+}\right)$, a direct calculation shows that

$$
\begin{aligned}
\int_{\mathbb{G}^{+}} \operatorname{div}_{\mathbb{G}}(f W)|u|^{p} d x & =-\int_{\mathbb{G}^{+}} f W \cdot \nabla_{\mathbb{G}}|u|^{p} d x \\
& =-p \int_{\mathbb{G}^{+}} f\left\langle W, \nabla_{\mathbb{G}} u\right\rangle|u|^{p-1} d x \\
& \leq p\left(\int_{\mathbb{G}^{+}}\left|\left\langle W, \nabla_{\mathbb{G}} u\right\rangle\right|^{p} d x\right)^{\frac{1}{p}}\left(\int_{\mathbb{G}^{+}}|f|^{\frac{p}{p-1}}|u|^{p} d x\right)^{\frac{p-1}{p}} .
\end{aligned}
$$

Here in the last line Hölder's inequality was applied. For $p>1$ and $q>1$ with $\frac{1}{p}+\frac{1}{q}=1$ recall Young's inequality

$$
a b \leq \frac{a^{p}}{p}+\frac{b^{q}}{q}, \text { for } a \geq 0, b \geq 0 .
$$

Let us set that 


$$
a:=\left(\int_{\mathbb{G}^{+}}\left|\left\langle W, \nabla_{\mathbb{G}} u\right\rangle\right|^{p} d x\right)^{\frac{1}{p}} \text { and } b:=\left(\int_{\mathbb{G}^{+}}|f|^{\frac{p}{p-1}}|u|^{p} d x\right)^{\frac{p-1}{p}} .
$$

By using Young's inequality in (2.15) and rearranging the terms, we arrive at

$$
\int_{\mathbb{G}^{+}}\left|\left\langle W, \nabla_{\mathbb{G}^{+}} u\right\rangle\right|^{p} d x \geq \int_{\mathbb{G}^{+}}\left(\operatorname{div}_{\mathbb{G}}(f W)-(p-1)|f|^{\frac{p}{p-1}}\right)|u|^{p} d x .
$$

We choose $W:=I_{i}$, which has the following form $I_{i}=(\overbrace{0, \ldots, 1}^{i}, \ldots, 0)$ and set

$$
f=\beta \frac{\left|\left\langle X_{i}(x), v\right\rangle\right|^{p-1}}{\operatorname{dist}\left(x, \partial \mathbb{G}^{+}\right)^{p-1}} .
$$

Now we calculate

$$
\begin{aligned}
\operatorname{div}_{\mathbb{G}}(W f) & =\left(\nabla_{\mathbb{G}} \cdot I_{i}\right) f=X_{i} f=\beta X_{i}\left(\frac{\left|\left\langle X_{i}(x), v\right\rangle\right|}{\operatorname{dist}\left(x, \partial \mathbb{G}^{+}\right)}\right)^{p-1} \\
& =\beta(p-1)\left(\frac{\left|\left\langle X_{i}(x), v\right\rangle\right|}{\operatorname{dist}\left(x, \partial \mathbb{G}^{+}\right)}\right)^{p-2} X_{i}\left(\frac{\left\langle X_{i}(x), v\right\rangle}{\operatorname{dist}\left(x, \partial \mathbb{G}^{+}\right)}\right) \\
& =\beta(p-1)\left(\frac{\left|\left\langle X_{i}(x), v\right\rangle\right|}{\operatorname{dist}\left(x, \partial \mathbb{G}^{+}\right)}\right)^{p-2}\left(\frac{X_{i}\left\langle X_{i}(x), v\right\rangle}{\operatorname{dist}\left(x, \partial \mathbb{G}^{+}\right)}-\frac{\left|\left\langle X_{i}(x), v\right\rangle\right|^{2}}{\operatorname{dist}\left(x, \partial \mathbb{G}^{+}\right)^{2}}\right) \\
& =\beta(p-1)\left[\left(\frac{\left|\left\langle X_{i}(x), v\right\rangle\right|}{\operatorname{dist}\left(x, \partial \mathbb{G}^{+}\right)}\right)^{p-2}\left(\frac{X_{i}\left\langle X_{i}(x), v\right\rangle}{\operatorname{dist}\left(x, \partial \mathbb{G}^{+}\right)}\right)-\frac{\left|\left\langle X_{i}(x), v\right\rangle\right|^{p}}{\operatorname{dist}\left(x, \partial \mathbb{G}^{+}\right)^{p}}\right],
\end{aligned}
$$

and

$$
|f|^{\frac{p}{p-1}}=|\beta|^{\frac{p}{p-1}} \frac{\left|\left\langle X_{i}(x), v\right\rangle\right|^{p}}{\operatorname{dist}\left(x, \partial \mathbb{G}^{+}\right)^{p}} .
$$

We also have

$$
\left\langle W, \nabla_{\mathbb{G}} u\right\rangle=(\overbrace{0, \ldots, 1}^{i}, \ldots, 0) \cdot\left(X_{1} u, \ldots, X_{i} u, \ldots, X_{N} u\right)^{T}=X_{i} u .
$$

Inserting the above calculations in (2.16) and summing over $i=1, \ldots, N$, we arrive at

$$
\begin{aligned}
\int_{\mathbb{G}^{+}} \sum_{i=1}^{N}\left|X_{i} u\right|^{p} d x \geq & -(p-1)\left(|\beta|^{\frac{p}{p-1}}+\beta\right) \int_{\mathbb{G}^{+}} \sum_{i=1}^{N} \frac{\left|\left\langle X_{i}(x), v\right\rangle\right|^{p}}{\operatorname{dist}\left(x, \partial \mathbb{G}^{+}\right)^{p}}|u|^{p} d x \\
& +\beta(p-1) \int_{\mathbb{G}^{+}} \sum_{i=1}^{N}\left(\frac{\left|\left\langle X_{i}(x), v\right\rangle\right|}{\operatorname{dist}\left(x, \partial \mathbb{G}^{+}\right)}\right)^{p-2} \frac{X_{i}\left\langle X_{i}(x), v\right\rangle}{\operatorname{dist}\left(x, \partial \mathbb{G}^{+}\right)}|u|^{p} d x .
\end{aligned}
$$

We complete the proof of Theorem 2.9. 
Remark 2.10 For $p \geq 2$, since

$$
\left|\nabla_{\mathbb{G}} u\right|^{p}=\left(\sum_{i=1}^{N}\left|X_{i} u\right|^{2}\right)^{\frac{p}{2}} \geq \sum_{i=1}^{N}\left(\left|X_{i} u\right|^{2}\right)^{\frac{p}{2}}
$$

we have the following inequality

$$
\begin{aligned}
\int_{\mathbb{G}^{+}}\left|\nabla_{\mathbb{G}} u\right|^{p} d x \geq & C_{2}(\beta, p) \int_{\mathbb{G}^{+}} \frac{\mathcal{W}_{p}(x)^{p}}{\operatorname{dist}\left(x, \partial \mathbb{G}^{+}\right)^{p}}|u|^{p} d x \\
& +\beta(p-1) \int_{\mathbb{G}^{+}} \sum_{i=1}^{N}\left(\frac{\left|\left\langle X_{i}(x), v\right\rangle\right|}{\operatorname{dist}\left(x, \partial \mathbb{G}^{+}\right)}\right)^{p-2} \frac{X_{i}\left\langle X_{i}(x), v\right\rangle}{\operatorname{dist}\left(x, \partial \mathbb{G}^{+}\right)}|u|^{p} d x .
\end{aligned}
$$

\section{Hardy inequalities on a convex domain of $\mathbb{G}$}

In this section, we present the geometric Hardy inequalities on the convex domains in stratified groups. The convex domain is understood in the sense of the Euclidean space. Let $\Omega$ be a convex domain of a stratified group $\mathbb{G}$ and let $\partial \Omega$ be its boundary. Below for $x \in \Omega$ we denote by $\nu(x)$ the unit normal for $\partial \Omega$ at a point $\hat{x} \in \partial \Omega$ such that $\operatorname{dist}(x, \Omega)=\operatorname{dist}(x, \hat{x})$. For the half-plane, we have the distance from the boundary $\operatorname{dist}(x, \partial \Omega)=\langle x, v\rangle-d$. As it is introduced in the previous section we also have the generalised angle function

$$
\mathcal{W}_{p}(x)=\left(\sum_{i=1}^{N}\left|\left\langle X_{i}(x), v\right\rangle\right|^{p}\right)^{\frac{1}{p}}
$$

with $\mathcal{W}(x):=\mathcal{W}_{2}(x)$

\subsection{Geometric $L^{2}$-Hardy inequality on a convex domain of $\mathbb{G}$}

Theorem 3.1 Let $\Omega$ be a convex domain of a stratified group $\mathbb{G}$. Then for $\beta<0$ we have

$$
\int_{\Omega}\left|\nabla_{\mathbb{G}} u\right|^{2} d x \geq C_{1}(\beta) \int_{\Omega} \frac{\mathcal{W}(x)^{2}}{\operatorname{dist}(x, \partial \Omega)^{2}}|u|^{2} d x+\beta \int_{\Omega} \sum_{i=1}^{N} \frac{X_{i}\left\langle X_{i}(x), v\right\rangle}{\operatorname{dist}(x, \partial \Omega)}|u|^{2} d x
$$

for all $u \in C_{0}^{\infty}(\Omega)$, and $C_{1}(\beta):=-\left(\beta^{2}+\beta\right)$. 
Proof of Theorem 3.1 We follow the approach of Simon Larson [11] by proving inequality (3.1) in the case when $\Omega$ is a convex polytope. We denote its facets by $\left\{\mathcal{F}_{j}\right\}_{j}$ and unit normals of these facets by $\left\{v_{j}\right\}_{j}$, which are directed inward. Then $\Omega$ can be constructed by the union of the disjoint sets $\Omega_{j}:=\left\{x \in \Omega: \operatorname{dist}(x, \partial \Omega)=\operatorname{dist}\left(x, \mathcal{F}_{j}\right)\right\}$. Now we apply the same method as in the case of the half-space $\mathbb{G}^{+}$for each element $\Omega_{j}$ with one exception that not all the boundary values are zero when we use the partial integration. As in the previous computation we have

$$
\begin{aligned}
0 \leq \int_{\Omega_{j}}\left|\nabla_{\mathbb{G}} u+\beta W u\right|^{2} d x= & \int_{\Omega_{j}} \sum_{i=1}^{N}\left|X_{i} u+\beta W_{i} u\right|^{2} d x \\
= & \int_{\Omega_{j}} \sum_{i=1}^{N}\left[\left|X_{i} u\right|^{2}+2 \operatorname{Re} \beta W_{i} u X_{i} u+\beta^{2} W_{i}^{2}|u|^{2}\right] d x \\
= & \int_{\Omega_{j}} \sum_{i=1}^{N}\left[\left|X_{i} u\right|^{2}+\beta W_{i} X_{i}|u|^{2}+\beta^{2} W_{i}^{2}|u|^{2}\right] d x \\
= & \int_{\Omega_{j}} \sum_{i=1}^{N}\left[\left|X_{i} u\right|^{2}-\beta\left(X_{i} W_{i}\right)|u|^{2}+\beta^{2} W_{i}^{2}|u|^{2}\right] d x \\
& +\beta \int_{\partial \Omega_{j}} \sum_{i=1}^{N} W_{i}\left\langle X_{i}(x), n_{j}(x)\right\rangle|u|^{2} d \Gamma_{\partial \Omega_{j}}(x),
\end{aligned}
$$

where $n_{j}$ is the unit normal of $\partial \Omega_{j}$ which is directed outward. Since $\mathcal{F}_{j} \subset \partial \Omega_{j}$ we have $n_{j}=-v_{j}$.

The boundary terms on $\partial \Omega$ vanish since $u$ is compactly supported in $\Omega$. So we only deal with the parts of $\partial \Omega_{j}$ in $\Omega$. Note that for every facet of $\partial \Omega_{j}$ there exists some $\partial \Omega_{l}$ which shares this facet. We denote by $\Gamma_{j l}$ the common facet of $\partial \Omega_{j}$ and $\partial \Omega_{l}$, with $\left.n_{k}\right|_{\Gamma_{j l}}=-\left.n_{l}\right|_{\Gamma_{j l}}$. From the above expression we get the following inequality

$$
\begin{aligned}
\int_{\Omega_{j}}\left|\nabla_{\mathbb{G}} u\right|^{2} d x \geq & \int_{\Omega_{j}} \sum_{i=1}^{N}\left[\left(\beta\left(X_{i} W_{i}\right)-\beta^{2} W_{i}^{2}\right)|u|^{2}\right] d x \\
& -\beta \int_{\partial \Omega_{j}} \sum_{i=1}^{N} W_{i}\left\langle X_{i}(x), n_{j}(x)\right\rangle|u|^{2} d \Gamma_{\partial \Omega_{j}}(x) .
\end{aligned}
$$

Now we choose $W_{i}$ in the form

$$
W_{i}(x)=\frac{\left\langle X_{i}(x), v_{j}\right\rangle}{\operatorname{dist}\left(x, \partial \Omega_{j}\right)}=\frac{\left\langle X_{i}(x), v_{j}\right\rangle}{\left\langle x, v_{j}\right\rangle-d},
$$

and a direct computation shows that

$$
X_{i} W_{i}(x)=\frac{X_{i}\left\langle X_{i}(x), v_{j}\right\rangle}{\operatorname{dist}\left(x, \partial \Omega_{j}\right)}-\frac{\left\langle X_{i}(x), v_{j}\right\rangle^{2}}{\operatorname{dist}\left(x, \partial \Omega_{j}\right)^{2}} .
$$

Inserting the expression (3.3) into inequality (3.2) we get

Birkhäuser 


$$
\begin{aligned}
& \int_{\Omega_{j}}\left|\nabla_{\mathbb{G}} u\right|^{2} d x \geq-\left(\beta^{2}+\beta\right) \int_{\Omega_{j}} \sum_{i=1}^{N} \frac{\left\langle X_{i}(x), v_{j}\right\rangle^{2}}{\operatorname{dist}\left(x, \partial \Omega_{j}\right)^{2}}|u|^{2} d x \\
& \quad+\beta \int_{\Omega_{j}} \sum_{i=1}^{N} \frac{X_{i}\left\langle X_{i}(x), v_{j}\right\rangle}{\operatorname{dist}\left(x, \partial \Omega_{j}\right)}|u|^{2} d x-\beta \int_{\Gamma_{j l}} \sum_{i=1}^{N} \frac{\left\langle X_{i}(x), v_{j}\right\rangle\left\langle X_{i}(x), n_{j l}\right\rangle}{\operatorname{dist}\left(x, \mathcal{F}_{j}\right)}|u|^{2} d \Gamma_{j l} .
\end{aligned}
$$

Now we sum over all partition elements $\Omega_{j}$ and let $n_{j l}=\left.n_{k}\right|_{\Gamma_{j l}}$, i.e. the unit normal of $\Gamma_{j l}$ pointing from $\Omega_{j}$ into $\Omega_{l}$. Then we get

$$
\begin{aligned}
\int_{\Omega}\left|\nabla_{\mathbb{G}} u\right|^{2} d x \geq & -\left(\beta^{2}+\beta\right) \int_{\Omega} \sum_{i=1}^{N} \frac{\left\langle X_{i}(x), v\right\rangle^{2}}{\operatorname{dist}(x, \partial \Omega)^{2}}|u|^{2} d x \\
& +\beta \int_{\Omega} \sum_{i=1}^{N} \frac{X_{i}\left\langle X_{i}(x), v\right\rangle}{\operatorname{dist}(x, \partial \Omega)}|u|^{2} d x \\
& -\beta \sum_{j \neq l} \int_{\Gamma_{j l}} \sum_{i=1}^{N} \frac{\left\langle X_{i}(x), v_{j}\right\rangle\left\langle X_{i}(x), n_{j l}\right\rangle}{\operatorname{dist}\left(x, \mathcal{F}_{j}\right)}|u|^{2} d \Gamma_{j l} \\
= & -\left(\beta^{2}+\beta\right) \int_{\Omega} \sum_{i=1}^{N} \frac{\left\langle X_{i}(x), v\right\rangle^{2}}{\operatorname{dist}(x, \partial \Omega)^{2}}|u|^{2} d x \\
& +\beta \int_{\Omega} \sum_{i=1}^{N} \frac{X_{i}\left\langle X_{i}(x), v\right\rangle}{\operatorname{dist}(x, \partial \Omega)}|u|^{2} d x \\
& -\beta \sum_{j<l} \int_{\Gamma_{j l}} \sum_{i=1}^{N} \frac{\left\langle X_{i}(x), v_{j}-v_{l}\right\rangle\left\langle X_{i}(x), n_{j l}\right\rangle}{\operatorname{dist}\left(x, \mathcal{F}_{j}\right)}|u|^{2} d \Gamma_{j l} .
\end{aligned}
$$

Here we used the fact that (by the definition) $\Gamma_{j l}$ is a set with $\operatorname{dist}\left(x, \mathcal{F}_{j}\right)=\operatorname{dist}\left(x, \mathcal{F}_{l}\right)$. From

$$
\Gamma_{j l}=\left\{x: x \cdot v_{j}-d_{j}=x \cdot v_{l}-d_{l}\right\}
$$

rearranging $x \cdot\left(v_{j}-v_{l}\right)-d_{j}+d_{l}=0$ we see that $\Gamma_{j l}$ is a hyperplane with a normal $v_{j}-v_{l}$. Thus, $v_{j}-v_{l}$ is parallel to $n_{j l}$ and one only needs to check that $\left(v_{j}-v_{l}\right) \cdot n_{j l}>0$. Observe that $n_{j l}$ points out and $v_{j}$ points into $j$ th partition element, so $v_{j} \cdot n_{j l}$ is non-negative. Similarly, we see that $v_{l} \cdot n_{j l}$ is non-positive. This means we have $\left(v_{j}-v_{l}\right) \cdot n_{j l}>0$. In addition, it is easy to see that

$$
\begin{aligned}
\left|v_{j}-v_{l}\right|^{2} & =\left(v_{j}-v_{l}\right) \cdot\left(v_{j}-v_{l}\right)=2-2 v_{j} \cdot v_{l} \\
& =2-2 \cos \left(\alpha_{j l}\right),
\end{aligned}
$$

which implies that

$$
\left(v_{j}-v_{l}\right) \cdot n_{j l}=\sqrt{2-2 \cos \left(\alpha_{j l}\right)},
$$

where $\alpha_{j l}$ is the angle between $v_{j}$ and $v_{l}$. So we obtain 


$$
\begin{aligned}
\int_{\Omega}\left|\nabla_{\mathbb{G}} u\right|^{2} d x \geq & -\left(\beta^{2}+\beta\right) \int_{\Omega} \sum_{i=1}^{N} \frac{\left\langle X_{i}(x), v\right\rangle^{2}}{\operatorname{dist}(x, \partial \Omega)^{2}}|u|^{2} d x \\
& +\beta \int_{\Omega} \sum_{i=1}^{N} \frac{X_{i}\left\langle X_{i}(x), v\right\rangle}{\operatorname{dist}(x, \partial \Omega)}|u|^{2} d x \\
& -\beta \sum_{j<l} \sum_{i=1}^{N} \int_{\Gamma_{j l}} \sqrt{1-\cos \left(\alpha_{j l}\right)} \frac{\left\langle X_{i}(x), n_{j l}\right\rangle^{2}}{\operatorname{dist}\left(x, \mathcal{F}_{j}\right)}|u|^{2} d \Gamma_{j l} .
\end{aligned}
$$

Here with $\beta<0$ and due to the boundary term signs we verify the inequality for the polytope convex domains.

Let us now consider the general case, that is, when $\Omega$ is an arbitrary convex domain. For each $u \in C_{0}^{\infty}(\Omega)$ one can always choose an increasing sequence of convex polytopes $\left\{\Omega_{j}\right\}_{j=1}^{\infty}$ such that $u \in C_{0}^{\infty}\left(\Omega_{1}\right), \Omega_{j} \subset \Omega$ and $\Omega_{j} \rightarrow \Omega$ as $j \rightarrow \infty$. Assume that $v_{j}(x)$ is the above map $v$ (corresponding to $\Omega_{j}$ ) we compute

$$
\begin{aligned}
\int_{\Omega}\left|\nabla_{\mathbb{G}} u\right|^{2} d x= & \int_{\Omega_{j}}\left|\nabla_{\mathbb{G}} u\right|^{2} d x \\
\geq & -\left(\beta^{2}+\beta\right) \int_{\Omega_{j}} \sum_{i=1}^{N} \frac{\left\langle X_{i}(x), v_{j}\right\rangle^{2}}{\operatorname{dist}\left(x, \partial \Omega_{j}\right)^{2}}|u|^{2} d x \\
& +\beta \int_{\Omega_{j}} \sum_{i=1}^{N} \frac{X_{i}\left\langle X_{i}(x), v_{j}\right\rangle}{\operatorname{dist}\left(x, \partial \Omega_{j}\right)}|u|^{2} d x \\
= & -\left(\beta^{2}+\beta\right) \int_{\Omega} \sum_{i=1}^{N} \frac{\left\langle X_{i}(x), v_{j}\right\rangle^{2}}{\operatorname{dist}\left(x, \partial \Omega_{j}\right)^{2}}|u|^{2} d x \\
& +\beta \int_{\Omega} \sum_{i=1}^{N} \frac{X_{i}\left\langle X_{i}(x), v_{j}\right\rangle}{\operatorname{dist}\left(x, \partial \Omega_{j}\right)}|u|^{2} d x \\
\geq & -\left(\beta^{2}+\beta\right) \int_{\Omega} \sum_{i=1}^{N} \frac{\left\langle X_{i}(x), v_{j}\right\rangle^{2}}{\operatorname{dist}(x, \partial \Omega)^{2}}|u|^{2} d x \\
& +\beta \int_{\Omega} \sum_{i=1}^{N} \frac{X_{i}\left\langle X_{i}(x), v_{j}\right\rangle}{\operatorname{dist}(x, \partial \Omega)}|u|^{2} d x
\end{aligned}
$$

Now we obtain the desired result when $j \rightarrow \infty$.

\section{2 $L^{p}$-Hardy's inequality on a convex domain of $\mathbb{G}$}

In this section we give the $L^{p}$-version of the previous results.

Theorem 3.2 Let $\Omega$ be a convex domain of a stratified group $\mathbb{G}$. Then for $\beta<0$ we have 


$$
\begin{aligned}
\int_{\Omega} \sum_{i=1}^{N}\left|X_{i} u\right|^{p} d x \geq & C_{2}(\beta, p) \int_{\Omega} \frac{\mathcal{W}_{p}(x)^{p}}{\operatorname{dist}(x, \partial \Omega)^{p}}|u|^{p} d x \\
& +\beta(p-1) \int_{\Omega} \sum_{i=1}^{N}\left(\frac{\left|\left\langle X_{i}(x), v\right\rangle\right|}{\operatorname{dist}(x, \partial \Omega)}\right)^{p-2}\left(\frac{X_{i}\left\langle X_{i}(x), v\right\rangle}{\operatorname{dist}(x, \partial \Omega)}\right)|u|^{p} d x,
\end{aligned}
$$

for all $u \in C_{0}^{\infty}(\Omega)$, and $C_{2}(\beta, p):=-(p-1)\left(|\beta|^{\frac{p}{p-1}}+\beta\right)$.

Proof of Theorem 3.2 Let us assume that $\Omega$ is the convex polytope as in the $p=2$ case. Thus, we consider the partition $\Omega_{j}$ as the previous case. For $f \in C^{1}\left(\Omega_{j}\right)$ and $W \in C^{\infty}\left(\Omega_{j}\right)$, a simple calculation shows that

$$
\begin{aligned}
\int_{\Omega_{j}} \operatorname{div}_{\mathbb{G}}(f W)|u|^{p} d x= & -p \int_{\Omega_{j}} f\left\langle W, \nabla_{\mathbb{G}} u\right\rangle|u|^{p-1} d x+\int_{\partial \Omega_{j}} f\left\langle W, n_{j}(x)\right\rangle|u|^{p} d \Gamma_{\partial \Omega_{j}}(x) \\
\leq & p\left(\int_{\Omega}\left|\left\langle W, \nabla_{\mathbb{G}} u\right\rangle\right|^{p} d x\right)^{\frac{1}{p}}\left(\int_{\Omega_{j}}|f|^{\frac{p}{p-1}}|u|^{p} d x\right)^{\frac{p-1}{p}} \\
& +\int_{\partial \Omega_{j}} f\left\langle W, n_{j}(x)\right\rangle|u|^{p} d \Gamma_{\partial \Omega_{j}}(x) .
\end{aligned}
$$

In the last line Hölder's inequality was applied. Recall again Young's inequality for $p>1, q>1$ and $\frac{1}{p}+\frac{1}{q}=1$, we have $a b \leq \frac{a^{p}}{p}+\frac{b^{q}}{q}$, for $a \geq 0, b \geq 0$. We now take $q:=\frac{p}{p-1}$ and

$$
a:=\left(\int_{\Omega}\left|\left\langle W, \nabla_{\mathbb{G}} u\right\rangle\right|^{p} d x\right)^{\frac{1}{p}} \text { and } \quad b:=\left(\int_{\Omega}|f|^{\frac{p}{p-1}}|u|^{p} d x\right)^{\frac{p-1}{p}} .
$$

By using Young's inequality in (3.6) and rearranging the terms, we arrive at

$$
\begin{aligned}
\int_{\Omega_{j}}\left|\left\langle W, \nabla_{\mathbb{G}} u\right\rangle\right|^{p} d x \geq & \int_{\Omega}\left(\operatorname{div}_{\mathbb{G}}(f W)-(p-1)|f|^{\frac{p}{p-1}}\right)|u|^{p} d x \\
& -\int_{\partial \Omega_{j}} f\left\langle W, n_{j}(x)\right\rangle|u|^{p} d \Gamma_{\partial \Omega_{j}}(x) .
\end{aligned}
$$

We choose $W:=I_{i}$ as a unit vector of the $i^{\text {th }}$ component and let

$$
f=\beta \frac{\left|\left\langle X_{i}(x), v_{j}\right\rangle\right|^{p-1}}{\operatorname{dist}\left(x, \mathcal{F}_{j}\right)^{p-1}} .
$$

As before a direct calculation shows that 


$$
\begin{aligned}
\operatorname{div}_{\mathbb{G}}(W f) & =X_{i} f=\beta X_{i}\left(\frac{\left|\left\langle X_{i}(x), v_{j}\right\rangle\right|}{\operatorname{dist}\left(x, \partial \mathcal{F}_{j}\right)}\right)^{p-1} \\
& =\beta(p-1)\left(\frac{\left|\left\langle X_{i}(x), v_{j}\right\rangle\right|}{\operatorname{dist}\left(x, \partial \mathcal{F}_{j}\right)}\right)^{p-2} X_{i}\left(\frac{\left\langle X_{i}(x), v_{j}\right\rangle}{\operatorname{dist}\left(x, \partial \mathcal{F}_{j}\right)}\right) \\
& =\beta(p-1)\left(\frac{\left|\left\langle X_{i}(x), v_{j}\right\rangle\right|}{\operatorname{dist}\left(x, \partial \mathcal{F}_{j}\right)}\right)^{p-2}\left(\frac{X_{i}\left\langle X_{i}(x), v_{j}\right\rangle}{\operatorname{dist}\left(x, \partial \mathcal{F}_{j}\right)}-\frac{\left|\left\langle X_{i}(x), v_{j}\right\rangle\right|^{2}}{\operatorname{dist}\left(x, \partial \mathcal{F}_{j}\right)^{2}}\right) \\
& =\beta(p-1)\left[\left(\frac{\left|\left\langle X_{i}(x), v_{j}\right\rangle\right|}{\operatorname{dist}\left(x, \partial \mathcal{F}_{j}\right)}\right)^{p-2}\left(\frac{X_{i}\left\langle X_{i}(x), v_{j}\right\rangle}{\operatorname{dist}\left(x, \partial \mathcal{F}_{j}\right)}\right)-\frac{\left|\left\langle X_{i}(x), v_{j}\right\rangle\right|^{p}}{\operatorname{dist}\left(x, \partial \mathcal{F}_{j}\right)^{p}}\right],
\end{aligned}
$$

and

$$
|f|^{\frac{p}{p-1}}=|\beta|^{\frac{p}{p-1}} \frac{\left|\left\langle X_{i}(x), v_{j}\right\rangle\right|^{p}}{\operatorname{dist}\left(x, \mathcal{F}_{j}\right)^{p}} .
$$

We also have

$$
\left\langle W, \nabla_{\mathbb{G}} u\right\rangle=(\overbrace{0, \ldots, 1}^{i}, \ldots, 0) \cdot\left(X_{1} u, \ldots, X_{i} u, \ldots, X_{N} u\right)^{T}=X_{i} u .
$$

Inserting the above calculations into (3.7) and summing over $i=\overline{1, N}$, we arrive at

$$
\begin{aligned}
\int_{\Omega_{j}} \sum_{i=1}^{N}\left|X_{i} u\right|^{p} d x \geq & -(p-1)\left(|\beta|^{\frac{p}{p-1}}+\beta\right) \int_{\Omega_{j}} \sum_{i=1}^{N} \frac{\left|\left\langle X_{i}(x), v_{j}\right\rangle\right|^{p}}{\operatorname{dist}\left(x, \partial \mathcal{F}_{j}\right)^{p}}|u|^{p} d x \\
& +\beta(p-1) \int_{\Omega_{j}} \sum_{i=1}^{N}\left(\frac{\left|\left\langle X_{i}(x), v_{j}\right\rangle\right|}{\operatorname{dist}\left(x, \partial \mathcal{F}_{j}\right)}\right)^{p-2}\left(\frac{X_{i}\left\langle X_{i}(x), v_{j}\right\rangle}{\operatorname{dist}\left(x, \partial \mathcal{F}_{j}\right)}\right)|u|^{p} d x \\
& -\beta \int_{\partial \Omega_{j}} \sum_{i=1}^{N}\left(\frac{\left|\left\langle X_{i}(x), v_{j}\right\rangle\right|}{\operatorname{dist}\left(x, \mathcal{F}_{j}\right)}\right)^{p-1}\left\langle X_{i}(x), n_{j}(x)\right\rangle|u|^{p} d \Gamma_{\partial \Omega_{j}}(x) .
\end{aligned}
$$

Now summing up over $\Omega_{j}$, and with the interior boundary terms we have 


$$
\begin{aligned}
\int_{\Omega} \sum_{i=1}^{N}\left|X_{i} u\right|^{p} d x \geq & -(p-1)\left(|\beta|^{\frac{p}{p-1}}+\beta\right) \sum_{i=1}^{N} \int_{\Omega} \frac{\left|\left\langle X_{i}(x), v\right\rangle\right|^{p}}{\operatorname{dist}(x, \partial \Omega)^{p}}|u|^{p} d x \\
& +\beta(p-1) \sum_{i=1}^{N} \int_{\Omega}\left(\frac{\left|\left\langle X_{i}(x), v\right\rangle\right|}{\operatorname{dist}(x, \partial \Omega)}\right)^{p-2}\left(\frac{X_{i}\left\langle X_{i}(x), v\right\rangle}{\operatorname{dist}(x, \partial \Omega)}\right)|u|^{p} d x \\
& -\beta \sum_{j \neq l} \sum_{i=1}^{N} \int_{\Gamma_{j l}}\left(\frac{\left|\left\langle X_{i}(x), v_{j}\right\rangle\right|}{\operatorname{dist}\left(x, \mathcal{F}_{j}\right)}\right)^{p-1}\left\langle X_{i}(x), n_{j l}(x)\right\rangle|u|^{p} d \Gamma_{j l} \\
= & -(p-1)\left(|\beta|^{\frac{p}{p-1}}+\beta\right) \sum_{i=1}^{N} \int_{\Omega} \frac{\left|\left\langle X_{i}(x), v\right\rangle\right|^{p}}{\operatorname{dist}(x, \partial \Omega)^{p}}|u|^{p} d x \\
& +\beta(p-1) \sum_{i=1}^{N} \int_{\Omega}\left(\frac{\left|\left\langle X_{i}(x), v\right\rangle\right|}{\operatorname{dist}(x, \partial \Omega)}\right)^{p-2}\left(\frac{X_{i}\left\langle X_{i}(x), v\right\rangle}{\operatorname{dist}(x, \partial \Omega)}\right)|u|^{p} d x \\
& -\beta \sum_{j<l} \sum_{i=1}^{N} \int_{\Gamma_{j l}}\left[\left(\frac{\left|\left\langle X_{i}(x), v_{j}\right\rangle\right|}{\operatorname{dist}\left(x, \mathcal{F}_{j}\right)}\right)^{p-1}\left\langle X_{i}(x), n_{j l}(x)\right\rangle\right. \\
& \left.-\left(\frac{\left|\left\langle X_{i}(x), v_{l}\right\rangle\right|}{\operatorname{dist}\left(x, \mathcal{F}_{l}\right)}\right)^{p-1}\left\langle X_{i}(x), n_{j l}(x)\right\rangle\right]|u|^{p} d \Gamma_{j l}
\end{aligned}
$$

As in the earlier case if the boundary term is positive we can discard it, so we want to show that

$$
\left[\left(\frac{\left|\left\langle X_{i}(x), v_{j}\right\rangle\right|}{\operatorname{dist}\left(x, \mathcal{F}_{j}\right)}\right)^{p-1}\left\langle X_{i}(x), n_{j l}(x)\right\rangle-\left(\frac{\left|\left\langle X_{i}(x), v_{l}\right\rangle\right|}{\operatorname{dist}\left(x, \mathcal{F}_{l}\right)}\right)^{p-1}\left\langle X_{i}(x), n_{j l}(x)\right\rangle\right] \geq 0 .
$$

Noting the fact that $n_{j l}=\frac{v_{j}-v_{l}}{\sqrt{2-2 \cos \left(\alpha_{j l}\right)}}$ and $\operatorname{dist}\left(x, \mathcal{F}_{j}\right)=\operatorname{dist}\left(x, \mathcal{F}_{l}\right)$ on $\Gamma_{j l}$, we arrive at

$$
\begin{aligned}
& \frac{1}{2-2 \cos \left(\alpha_{j l}\right)}\left[\left(\frac{\left|\left\langle X_{i}(x), v_{j}\right\rangle\right|}{\operatorname{dist}\left(x, \mathcal{F}_{j}\right)}\right)^{p-1}\left\langle X_{i}(x), v_{j}-v_{l}\right\rangle-\left(\frac{\left|\left\langle X_{i}(x), v_{l}\right\rangle\right|}{\operatorname{dist}\left(x, \mathcal{F}_{l}\right)}\right)^{p-1}\left\langle X_{i}(x), v_{j}-v_{l}\right\rangle\right] \\
& =\frac{\left|\left\langle X_{i}(x), v_{j}\right\rangle\right|^{p}-\left|\left\langle X_{i}(x), v_{j}\right\rangle\right|^{p-1}\left\langle X_{i}(x), v_{l}\right\rangle-\left|\left\langle X_{i}(x), v_{l}\right\rangle\right|^{p-1}\left\langle X_{i}(x), v_{j}\right\rangle+\left|\left\langle X_{i}(x), v_{l}\right\rangle\right|^{p}}{\left(2-2 \cos \left(\alpha_{j l}\right)\right) \operatorname{dist}\left(x, \mathcal{F}_{j}\right)^{p-1}} \\
& =\frac{\left(\left|\left\langle X_{i}(x), v_{j}\right\rangle\right|-\left|\left\langle X_{i}(x), v_{l}\right\rangle\right|\right)\left(\left|\left\langle X_{i}(x), v_{j}\right\rangle\right|^{p-1}-\left|\left\langle X_{i}(x), v_{l}\right\rangle\right|^{p-1}\right)}{\left(2-2 \cos \left(\alpha_{j l}\right)\right) \operatorname{dist}\left(x, \mathcal{F}_{j}\right)^{p-1}} \geq 0 .
\end{aligned}
$$

Here we have used the equality $(a-b)\left(a^{p-1}-b^{p-1}\right)=a^{p}-a^{p-1} b-b^{p-1} a+b^{p-1}$ with $a=\left|\left\langle X_{i}(x), v_{j}\right\rangle\right|$ and $b=\left|\left\langle X_{i}(x), v_{l}\right\rangle\right|$. From the above expression we note that the boundary term in $\Omega$ is positive and $\beta<0$. By discarding the boundary term we complete the proof.

Remark 3.3 For $p \geq 2$, since 


$$
\left|\nabla_{\mathbb{G}} u\right|^{p}=\left(\sum_{i=1}^{N}\left|X_{i} u\right|^{2}\right)^{\frac{p}{2}} \geq \sum_{i=1}^{N}\left(\left|X_{i} u\right|^{2}\right)^{\frac{p}{2}},
$$

we have the following inequality

$$
\begin{aligned}
\int_{\Omega}\left|\nabla_{\mathbb{G}} u\right|^{p} d x \geq & C_{2}(\beta, p) \int_{\Omega} \frac{\mathcal{W}_{p}(x)^{p}}{\operatorname{dist}(x, \partial \Omega)^{p}}|u|^{p} d x \\
& +\beta(p-1) \int_{\Omega} \sum_{i=1}^{N}\left(\frac{\left|\left\langle X_{i}(x), v\right\rangle\right|}{\operatorname{dist}(x, \partial \Omega)}\right)^{p-2}\left(\frac{X_{i}\left\langle X_{i}(x), v\right\rangle}{\operatorname{dist}(x, \partial \Omega)}\right)|u|^{p} d x .
\end{aligned}
$$

Acknowledgements The first author was supported by the EPSRC Grant EP/R003025/1, by the Leverhulme Research Grant RPG-2017-151, and by the FWO Odysseus Grant. The second author was supported in parts by the Nazarbayev University Grant N090118FD5342. Third author was supported by the Nazarbayev University program 091019CRP2120 and in parts by the Nazarbayev University Grant 240919FD3901.

Open Access This article is licensed under a Creative Commons Attribution 4.0 International License, which permits use, sharing, adaptation, distribution and reproduction in any medium or format, as long as you give appropriate credit to the original author(s) and the source, provide a link to the Creative Commons licence, and indicate if changes were made. The images or other third party material in this article are included in the article's Creative Commons licence, unless indicated otherwise in a credit line to the material. If material is not included in the article's Creative Commons licence and your intended use is not permitted by statutory regulation or exceeds the permitted use, you will need to obtain permission directly from the copyright holder. To view a copy of this licence, visit http://creativecommons.org/licen ses/by/4.0/.

\section{References}

1. Ancona, A.: On strong barriers and an inequalities of Hardy for domains $\$\{\{\backslash \operatorname{mathbb}\{\mathrm{R}\}\}\}^{\wedge} \mathrm{n} \$$. J. Lond. Math. Soc. 43, 274-290 (1986)

2. Avkhadiev F.G., Laptev A.: On a sharp Hardy inequality for convex domains. International Mathematical Series 12. Around the Research of Vladimir Maz'ya I, pp. 1-12. Springer, New York (2010)

3. Avkhadiev, F.G., Wirths, K.J.: Unified Poincare and Hardy inequalities with sharp constants for convex domains. ZAMM Z. Angew. Math. Mech. 87, 632-642 (2007)

4. Bonfiglioli, A., Lanconelli, E., Uguzzoni, F.: Stratified Lie Groups and Potential Theory for their Sub-Laplacians. Springer, Berlin (2007)

5. D'Ambrozio, L.: Some Hardy inequalities on the Heisenberg group. Differ. Uravn. 40, 509-521 (2004)

6. Davies E.B.: A review of Hardy Inequalities. The Maz'ya anniversary Collection, vol 2. Oper. Theory Adv. Appl., pp. 55-67 (1999)

7. Danielli, D., Garofalo, N., Nhieu, D.M.: Sub-Riemannian calculus on hypersurfaces in Carnot groups. Adv. Math. 215, 292-378 (2007)

8. Fischer V., Ruzhansky M.: Quantization on Nilpotent Lie Groups. Progress in Mathematics, vol. 314, Birkhäuser (2016) (open access book)

9. Folland G.B., Stein E.M.: Hardy Spaces on Homogeneous Groups. Mathematical Notes, vol. 28. Princeton University Press, Princeton. University of Tokyo Press, Tokyo (1982)

10. Garofalo, N.: Geometric second derivative estimates in Carnot groups and convexity. Manuscr. Math. 126, 353-373 (2008) 
11. Larson, S.: Geometric Hardy inequalities for the sub-elliptic Laplacian on convex domain in the Heisenberg group. Bull. Math. Sci. 6, 335-352 (2016)

12. Luan, J.W., Yang, Q.H.: A Hardy type inequality in the half-space on $\$\{\{\backslash \mathrm{mathbb}\{\mathrm{R}\}\}\}^{\wedge} \mathrm{n} \$$ and Heisenberg group. J. Math. Anal. Appl. 347, 645-651 (2008)

13. Opic, B., Kufner, A.: Hardy-Type Inequalities. Pitman Research Notes in Mathematics Series, vol. 219 (1990)

14. Ruzhansky, M., Suragan, D.: Layer potentials, Kac's problem, and refined Hardy inequality on homogeneous Carnot groups. Adv. Math. 308, 483-528 (2017) 